\title{
Human Direct Interface
}

\section{Luis Miguel Muñoz*}

Automatic Control and Computer Engineering Department, Universitat Poletecnica de Catalunya, Spain

Electronic devices surround us in a continuous and incessant way, either to make life more comfortable, safer or performing new ways of behaving leisure or work. The interface to these devices has evolved to simplify their use making it more intuitive and closer to the user, but is restricted to the use of fingers and/or hands (and maybe voice) as input information channel from human to the systems, and eye and/or ear as output channel (haptic feedback including the fingers or hands are also elements of output) from the systems to the human.

The continuous use of portable devices such as smartphones, tablets and notebooks tend to isolate users from the surrounding environment, including people with whom we interact, in order to receive information about products or services and performing tasks in digital format. Ergonomics in the use of these interfaces must provide adequate security and comfort as they evolve. But technology will continue to evolve in this way? Will we continue watching at small screens and activating buttons or tactile elements?
The evolution of human intelligence is associated with the use of hands to build and work with tools, interact with them in the environment has been the most direct, efficient and effective throughout history. However the actions taken with hands are only a reflection of motor commands from the brain. Would it benefit in this regard a direct interface with the information and systems?

Brain-machine interfaces use sensors to obtain EEG signals to convert them into orders of movement or action. Although at a primitive stage, certain commercial devices are used to interface with computers as a pointing device or control for some videogames. The development of these devices solve the input to the machine or virtual worlds, and can be used to turn on/off appliances, lights, air conditioning or change channels on the TV. In more advanced stages, articulated robots can be operated, entering text or driving vehicles, all them only with the intention to do it. But, what happens with the collection of information? How to dispense with the visual/auditory channel? This will be another history.
*Corresponding author: Luis Miguel Muñoz, Department of Automatic Contro and Computer Engineering, Universitat Politècnica de Catalunya, Spain, E-mail: luis.miguel.munoz@upc.edu

Received December 18, 2011; Accepted December 20, 2011; Published December 20, 2011

Citation: Muñoz LM (2011) Human Direct Interface. J Ergonom 1:e105. doi:10.4172/2165-7556.1000e105

Copyright: (c) 2011 Muñoz LM. This is an open-access article distributed under the terms of the Creative Commons Attribution License, which permits unrestricted use, distribution, and reproduction in any medium, provided the original author and source are credited. 